\title{
Body schematics: On the role of the body schema in embodied lexical-semantic representations
}

\author{
Shirley-Ann Rueschemeyer ${ }^{\mathrm{a}, *}$, Christian Pfeiffer ${ }^{\mathrm{a}, \mathrm{b}}$, Harold Bekkering ${ }^{\mathrm{a}}$ \\ a Radboud University, Donders Institute for Brain, Cognition and Behavior, Nijmegen, The Netherlands \\ ${ }^{\mathrm{b}}$ Freie Universität Berlin, Berlin, Germany
}

\section{A R T I C L E I N F O}

\section{Article history:}

Received 20 April 2009

Received in revised form 11 August 2009

Accepted 14 September 2009

Available online 24 September 2009

\section{Keywords:}

Embodiment

Semantics

Action

Body schema

Sense of body

\begin{abstract}
A B S T R A C T
Words denoting manipulable objects activate sensorimotor brain areas, likely reflecting action experience with the denoted objects. In particular, these sensorimotor lexical representations have been found to reflect the way in which an object is used. In the current paper we present data from two experiments (one behavioral and one neuroimaging) in which we investigate whether body schema information, putatively necessary for interacting with functional objects, is also recruited during lexical processing. To this end, we presented participants with words denoting objects that are typically brought towards or away from the body (e.g., cup or key, respectively). We hypothesized that objects typically brought to a location on the body (e.g., cup) are relatively more reliant on body schema representations, since the final goal location of the cup (i.e., the mouth) is represented primarily through posture and body coordinates. In contrast, objects typically brought to a location away from the body (e.g., key) are relatively more dependent on visuo-spatial representations, since the final goal location of the key (i.e., a keyhole) is perceived visually. The behavioral study showed that prior planning of a movement along an axis towards and away from the body facilitates processing of words with a congruent action semantic feature (i.e., preparation of movement towards the body facilitates processing of cup.). In an fMRI study we showed that words denoting objects brought towards the body engage the resources of brain areas involved in the processing information about human bodies (i.e., the extra-striate body area, middle occipital gyrus and inferior parietal lobe) relatively more than words denoting objects typically brought away from the body. The results provide converging evidence that body schema are implicitly activated in processing lexical information.
\end{abstract}

(c) 2009 Elsevier Ltd. All rights reserved.

\section{Introduction}

Recently, embodied theories of language processing have put forth the proposal that brain areas involved in perception and action are also recruited during language processing (Barsalou, 2008; Fischer \& Zwaan, 2008; Pulvermüller, 2001). For example, words and sentences denoting actions (e.g., grasp, run) or objects highly associated with actions (e.g., cup, hammer) have been shown to elicit activation in brain areas relevant for action planning and execution (Beauchamp \& Martin, 2007; Chao \& Martin, 2000), while language materials denoting objects highly associated with a specific smell (e.g., garlic or cinnamon) activate olfactory cortex, relevant for processing smells (Gonzalez et al., 2006).

In particular, action-related objects selectively activate a network of neural areas including the ventral premotor cortex (vPMC),

\footnotetext{
* Corresponding author at: Donders Centre for Brain, Cognition and Behaviour, Centre for Cognition, Montessorilaan 3, 6525 HR, Nijmegen, The Netherlands.

E-mail address: s.rueschemeyer@donders.ru.nl (S.-A. Rueschemeyer).
}

inferior parietal cortex, posterior lateral temporal cortex and medial temporal cortex (Beauchamp \& Martin, 2007; Chao \& Martin, 2000; Saccuman et al., 2006; but see also Assmus, Giessing, Weiss, \& Fink, 2007). These sensorimotor lexical representations have been shown to be highly sensitive to the way in which an object is used functionally. For example, although both the words cup and bookend denote man-made tools that can be easily hand-held, cup elicits greater levels of activation in action-relevant areas than bookend, presumably because one must move a cup continuously (in contrast to a bookend) to use it functionally (Rueschemeyer et al., in press; see also Bub, Masson, \& Cree, 2008; Masson, Bub, \& Newton-Taylor, 2008 for converging behavioral evidence). These results show that how an object is typically manipulated is critical in determining how the lexical representation of the object is processed in the brain, and further that embodied lexical representations are quite specific in the type of experiential information they reflect.

In the current paper we present data from two experiments (one behavioral and one neuroimaging) in which we investigate whether body schematic configurations, known to be critical for 
interactions with functional objects, are also recruited during lexical-semantic processing. The body schema is an on-line representation of the body in terms of posture and its extension in space (Head \& Holmes, 1911; Holmes \& Spence, 2004). It dynamically maps the current positions of body parts in relation to one another and interacts with motor systems to support action planning and execution (e.g., Parsons, 1994). Interestingly the body schema has been shown to temporarily extend beyond the physical realms of the body when tools are used (Iriki, Tanaka, \& I, 1996; Maravita, Spence, \& Driver, 2003; Maravita \& Iriki, 2004). In other words, during tool-use, the body schema appears to become extended to incorporate physical attributes (e.g., length) of the tool. The tip of the tool, or the part of the tool which will create an action effect, becomes particularly relevant for these plastic extensions (Maravita \& Iriki, 2004). Because both the physical extension (e.g. shape, length) and action-effector (e.g., distal part for a hammer, proximal part for hairspray) vary for different tools, it has been suggested that different tools extend the body schema in different ways (Arbib et al., 2009). This is particularly relevant for looking at the role of body schema in action planning, as action plans are known to rely heavily on action goals (Rosenbaum, Loukopoulos, Meulenbroek, Vaughan, \& Englebrecht, 1995).

Clearly, different tools will extend and make use of body schema information in different ways. While a cup and a key may both extend the physical length of the hand (i.e., extend peripersonal space), the action goals of cups and keys are very different, and make very different demands on action planning. In preparing to use a cup, the user becomes implicitly aware of where the mouth is in relation to the hand, thus utilizing the body schema to guide one effector (the incorporated cup) to another (the mouth). In preparing to use a key, on the other hand, the user must visually detect the keyhole, and bring the hand holding to key to this location in peripersonal space, thereby creating an action plan with less reliance on the body schema than seen in the example of the cup. Embodied theories of language processing, as pointed out previously, state that internal simulations of actual interactions with functional objects (i.e., tools) are reflected in lexical-semantic representations. Thus in the current studies, we investigated whether different reliance on body schema (i.e., a representational system underlying action planning) in planning these real-world interactions are also reflected in lexical-semantic representations.

To this end, we presented participants with words denoting objects that are typically brought towards or away from the body when used (e.g., cup or key, respectively). In a behavioral study we tested the hypothesis that words denoting these different types of objects are associated with specific movement parameters. Previous research has shown that lexical and sentential meaning can modulate speed of response in action execution (e.g., Boulenger et al., 2006; Glenberg \& Kaschak, 2002). Specifically, Glenberg and Kaschak (2002) introduced the action-sentence compatibility effect (ACE), which describes the observation that participants are faster to execute an action that is congruent rather than incongruent to the movement implied by a previous sentence (e.g., movement of hand towards body in response to the sentence "open the drawer" is faster than movement of the hand away from the body). This suggests (1) that action and language share neural resources, but also that (2) specific action parameters (i.e., movement in a specific direction), and not simply action in general is relevant for language representations (see also Tucker \& Ellis, 2004). In the current study we investigated whether the ACE effect could be used to dissociate between single words denoting manipulable objects used with different action trajectories (i.e., towards vs. away from the body).

In addition to being associated with different movement parameters, we suggest that objects typically brought to a location on the body (e.g., cup) are relatively more reliant on body schema, since the final goal location of the cup (i.e., the mouth) is represented pri- marily through posture and body co-ordinates. In contrast, objects typically brought to a location away from the body (e.g., key) are relatively more dependent on visuo-spatial representations, since the final goal location of the key (i.e., a keyhole) is perceived visually. We tested this hypothesis in a neuroimaging experiment, in which we investigated whether brain areas known to support body schema are increasingly active when participants process words with putatively more body information (e.g., cup > key).

\section{Behavioral study}

\subsection{Experimental methods}

\subsubsection{Participants}

Nineteen right-handed, native Dutch students of the Radboud University participated in the study. All participants had normal or corrected to normal vision and no history of neurological disorders. Beforehand all participants were informed about the experimental procedures, were given practice trials and signed informed consent. Afterwards all students were awarded monetarily or with course credit for participating.

\subsubsection{Stimuli}

A total of 100 lexical stimuli were created for the experiment. 80 of the total 100 stimuli were real Dutch words, and comprised the critical experimental stimuli; the remaining 20 stimuli were Dutch pseudowords (PW) (i.e., phonotactically and orthographically legal letter strings with no meaning in Dutch) and served as filler items and catch trials (see procedures below). Critical stimuli belonged to one of two experimental conditions: (1) words denoting objects whose functional use requires movement towards a goal location on the body (Body Words: BW) or (2) words denoting objects whose functional use requires movement away from the body towards a goal location in the surrounding environment (World Words: WW). All denoted objects were thus functionally manipulable objects (i.e., they require manipulation in order to function), but they differ with respect to the direction of movement typically associated with their functional use. BW are brought towards the body for functional use (e.g., cup, comb), whereas WW are brought away from the body for functional use (e.g., key, screwdriver).

The 80 critical word stimuli were matched across word conditions (BW, WW) for word length (BW: mean $=6.7$ letters, $S D=2.6$; $W W$ : mean $=6.3, S D=2.04$ ), frequency of use according to CELEX (BW: mean $=569, \mathrm{SD}=128$; WW: mean $=513$, $\mathrm{SD}=118$ ) and imageability according to participants' own ratings on a scale of 1-7 (BW: mean $=6.53, \mathrm{SD}=0.34 ; \mathrm{WW}$ : mean $=6.50, \mathrm{SD}=0.50$ ). Independent sample $t$ tests showed that values for the two-word group did not differ significantly in any of these parameters (all $p s>0.1$ )

\subsubsection{Procedure}

Stimulus words were presented as white letter strings with a font size of 36 in Arial Style on a black background. Stimuli were presented on a 19 in. display with a resolution of $800 \times 600$ pixels and a refresh rate of $100 \mathrm{~Hz}$. The viewing distance was approximately $70 \mathrm{~cm}$ resulting a visual angle of about $13^{\circ}$ (for the largest lexical letter string $=12$ letters). Participants gave responses by button press with the index finger of the right hand. A custom-built button box on which three equidistant buttons were mounted served as response device.

Participants were seated comfortably in front of a computer monitor, with the button box in front of them. The button box contained 3 equidistant buttons arranged linearly in front of the participant such that participants had a far, middle and a near button. Each trial was initiated by the participant pressing the middle button. Participants were instructed to keep the middle button depressed until giving their final response (see further below). With the middle button depressed, every trial followed the same structure: first, a fixation cross was presented in the center of the screen for $400 \mathrm{~ms}$. This was followed directly by an action cue (the letter A or B) which was shown for $500 \mathrm{~ms}$, and which instructed participants as to which of the two other buttons on the button box they should press in response to the target word stimulus. For half of the participants the cue A indicated that the participant should move from the middle button to the button farthest away from them on the button box (i.e., away movement), while the cue B indicated that a movement should be made from the middle button to the closest of the three buttons (i.e., towards movement). For the other half of the participants the opposite was true. Following the action cue, participants saw a second fixation cross for $1000 \mathrm{~ms}$, and then finally the target word. Participants were instructed to respond to the target word stimulus using the previously cued action if the word stimulus was a real Dutch word. In the event of a pseudoword participants were instructed to withhold the prepared response. Participants thus saw an action cue for all trials, but were required to respond only to trials in which the target word was a real word. Target words remained visible until participants responded, or for a maximum of $3000 \mathrm{~ms}$. No feedback was provided during the experiment. The differences between the experimental procedure adopted here and that introduced initially by Glenberg and Kaschak (2002) were motivated primarily by two factors: (1) by having participants prepare responses before seeing word stimuli, action preparation served as 
Table 1

Mean response times (reaction times and movement times in milliseconds), and performance rates (overall percent correct, rate of misses and rate of incorrect movements) and SE for participants responding to Body Words and World Words after preparing a motion towards or away from the body.

\begin{tabular}{|c|c|c|c|c|}
\hline & \multicolumn{2}{|l|}{ Body Words } & \multicolumn{2}{|l|}{ World Words } \\
\hline Reaction time & $633(21)$ & $659(29)$ & $661(31)$ & $639(26)$ \\
\hline Performance rate & $99.72(0.28)$ & $99.17(0.45)$ & $98.06(0.82)$ & $98.61(0.68)$ \\
\hline Rate of misses & 0 & 0 & $0.56(0.38)$ & $0.27(0.28)$ \\
\hline Rate of incorrect movements & $0.28(0.28)$ & $0.83(0.45)$ & $1.39(0.79)$ & $1.11(0.65)$ \\
\hline
\end{tabular}

a prime in the current experiment, allowing inferences about the directionality of action-language interactions to be drawn. Secondly, by presenting participants with varying action cues in each trial, we could use a fully randomized within-subjects design in contrast to the blocked design used by Glenberg and Kaschak (2002).

Participants were presented with a total of 100 word stimuli belonging to the three conditions described above (BW, WW, PW). The order of stimulus presentation was randomized individually for each participant. All participants saw all stimuli. In half of the trials for each real word condition (BW, WW) participants were cued to make a movement towards the body and in the other half a movement away from the body. We thus used a $2 \times 2$ factorial design with the within-subjects factors Word Meaning (BW, WW) and Response (Towards, Away).

\subsubsection{Results}

Performance rates (percentage of trials correctly responded to) and response times constituting both reaction times (i.e., time between presentation of the word and release of the middle button) and movement times (i.e., the time between releasing the middle button and depression of the response button) can be seen in Table 1. In all statistical tests, a Type I error rate of $\alpha=0.05$ was used. Data from one participant was excluded from the final analysis, as she had excessively long reaction times (mean over $1.5 \mathrm{~s}$ in all conditions), leaving the data from 18 participants in the final analysis.

\subsubsection{Performance dato}

Performance data was analyzed along two lines in each trial. First, accuracy rates for the lexical decision task (i.e., whether or not participants released middle button upon seeing a word stimulus) indicated how well participants distinguished between words and pseudowords. These errors were labeled 'misses'. Secondly, accuracy rates for the direction of response indicated whether participants successfully followed the instructions given by the movement cue on any given trial. These errors were labeled 'incorrect movements'. Error rates for both types of error were subjected to a repeated measures multiple analysis of variance (MANOVA) with the within-subjects factors Word Meaning (Body, World) and Response (Towards, Away).

Participants made a few misses overall ( $0.21 \%$ errors), indicating that they were capable of detecting all critical word stimuli as real words. The MANOVA showed no significant differences in the number of misses made between levels of Word Meaning $(F(1,17)=3.4 ; p>0.05)$ or Response $(F(1,17)=0.32, p>0.1)$. There was also no significant interaction between Word Meaning $\times$ Response $(F(1,17)=0.32, p>0.1)$.

Participants also made few incorrect movements overall ( $0.90 \%$ errors), indicating that they were indeed following the instructions given by the movement cue. The results of the MANOVA again showed no significant differences in the number of incorrect movements made between levels of Word Meaning $(F(1,17)=1.73$, $p>0.1)$ or Response $(F(1,17)=0.06, p>0.1)$. There was also no significant interaction between Word Meaning $\times$ Response $(F(1,17)=0.45, p>0.1)$

\subsubsection{Response times}

Responses to all trials were recorded and outliers $(3 \times \mathrm{STD} \pm$ mean RT $)$ as well as trials in which participants made an incorrect response (see above) were excluded ( $1.95 \%$ of total data). Mean reaction times (i.e., the time between presentation of the word and release of the middle button) and movement times (i.e., the time between releasing the middle button and depression of the response button) were calculated for each participant in each condition. A MANOVA was calculated with the withinsubjects factors Word Meaning (Body, World) and Response (Towards, Away) and the two dependent variables reaction time and movement time.

Reaction times: Participants did not find recognition of one category of words or execution of one motion parameter more difficult than the other, as indicated by the lack of any significant main effects in reaction times (Word Meaning: $F(1,17)=0.15$, $p>0.10$, Response: $F(1,17)=0.06, p>0.1)$. However, participants were faster to recognize words with specific meanings having prepared a specific movement, as indicated by a significant Word Meaning $\times$ Response interaction $(F(1,17)=5.07$, $p<0.05$, partial $\eta^{2}=0.23$ ).

Resolution of the Word Meaning $\times$ Response interaction showed that participants were indeed faster to respond to trials in which Word Meaning and Response were congruent (i.e., Body-Towards $=$ BT, World-Away $=$ WA) than incongruent (i.e., Body-Away $=$ BA and World-Towards $=$ WT $)($ Congruent Trials: mean $=636, S E=23$,
Incongruent Trials: mean $=660, \mathrm{SE}=30$ ). This was also reflected in the planned post hoc comparisons (one-sided $t$-tests) of RTs for the two Response types within each level of Word Meaning. For Body Words participants were faster to respond to words after preparing a Towards rather than an Away movement (BT: mean $=633, \mathrm{SE}=21$ $\mathrm{BA}$ : mean $=659, \mathrm{SE}=29, t(17)=2.17, p<0.05)$. For World Words participants showed a tendency to respond more quickly if an action had been prepared Away rather than Towards the body (WA: mean $=639, \mathrm{SE}=27$, WT: mean $=661, \mathrm{SE}=31, t(17)=1.55$, $p=0.07)$

Movement times: No significant main effects or interactions were observed in movement times, although a tendency for slower movement times for movements towards the body was observed (Word: $F(1,17)=0.03, p>0.1$; Response $F(1,17)=3.04, p=0.1$; Word $\times$ Response: $F(1,17)=1.58, p>0.1)$.

\subsubsection{Discussion}

The results of the behavioral study show a clear congruency effect between word meaning and action preparation. Specifically, participants were faster to respond to words denoting objects typically brought towards the body to use (e.g., cup, comb), if they had prepared a hand motion towards the body. Likewise, participants were faster to respond to words denoting objects typically brought away from the body and towards another object in the environment for use (e.g., key, screwdriver), if they had prepared a hand motion away from the body.

These results are consistent with a number of studies reporting modulating effects of action preparation and execution on language processing. For example, Glenberg and Kaschak (2002) showed that participants are faster to make an active response when the direction of motion described in a sentence matches the direction of motion required to make the response (see also Borreggine \& Kaschak, 2006) Likewise, Zwaan and Taylor (2006) showed that sentences describing manual rotation (e.g., she turned the volume up/down) facilitate a congruent manual rotation of the hand (e.g., rotation of a knob to the right/left). In the current study words rather than entire sentences were used, however the underlying principle remains the same. Indeed previous research has shown that single words, as well as sentences are highly associated with actions (Myung, Blumstein, \& Sedivy, 2006).

The results of the behavioral study indicate that our word stimuli are indeed highly associated with specific actions (i.e., Body Words are associated with a hand action towards the body, while World Words are associated with a hand action away from the body). In a follow-up fMRI study we tested the hypothesis that the different nature of these actions may result in different neural correlates. Specifically, we suggest that objects typically brought to a location on the body (e.g., cup) are relatively more reliant on body schema representations, since the final goal location of the cup (i.e., the mouth) is represented primarily through posture and body co-ordinates. In contrast, objects typically brought to a location away from the body (e.g., hammer) are relatively more dependent on visuo-spatial representations, since the final goal location of the hammer (i.e., a nail) is perceived visually.

\section{Functional MRI study}

\subsection{Experimental methods}

\subsubsection{Participants}

Eighteen native Dutch speakers ( 11 females, mean age $=23.7$ years, $S D=3.1$ years) participated in the fMRI study. All participants had normal or corrected to normal vision and no history of neurological disorders. Beforehand all participants were informed about the experimental procedures, were given practice trials and signed informed consent. Afterwards all students were awarded 10 euros for participating.

\subsubsection{Stimuli}

A total of 200 word stimuli were created for the experiment. 160 of the total 200 stimuli were real Dutch words, and comprised the critical experimental stimuli (see Appendix B); the remaining 40 stimuli were Dutch pseudowords (i.e., phonotactically and orthographically legal letter strings with no meaning in Dutch) and served as filler items and catch trials (see procedures below). As in the behavioral study, critical stimuli belonged to one of two experimental conditions: (1) words denoting objects whose functional use requires movement towards a goal location on the body (Body Words: BW) or (2) words denoting objects whose functional use 
requires movement away from the body towards a goal location in the surrounding environment (World Words: WW). In addition to the differences in the movement parameters associated with BW and WW, the action-target (i.e., the location to which the object is brought during functional use) is fundamentally different for BW and WW stimuli. Specifically, BW objects are brought towards the body to act on a location on the body (e.g., cup to mouth, or comb to head), which is not usually seen by the actor, and which is potentially represented in a postural rather than visual co-ordinate system. WW objects, on the other hand, are brought away from the body to act on a location in the surrounding environment (e.g., key to lock, or screwdriver to screw), which is generally perceived visually by the actor, and may thus rely more heavily on visual rather than postural co-ordinates.

The 160 critical word stimuli were matched for word length (BW: mean $=6.64$ letters, $\mathrm{SD}=2.05$; $\mathrm{WW}$ : mean $=6.11, \mathrm{SD}=2.04$ ), frequency as rated by the participants on a scale of 1-7 in a questionnaire administered after the scanner session (BW: mean $=4.37, \mathrm{SD}=1.37 ; \mathrm{WW}$ : mean $=4.28, \mathrm{SD}=1.15$ ) and imageability as rated by the participants on a scale of $1-7$ on the post-scanning questionnaire (BW: mean $=6.61, \mathrm{SD}=0.36$; WW: mean $=6.57, \mathrm{SD}=0.38$ ). Independent sample $t$-tests comparing length, frequency and imageablility scores between the two word conditions revealed no significant effects (all $p s>0.1$ ). In addition, participants were asked to give a rating for all words reflecting their action association to the word. Specifically, participants were asked to rate on a score of 1-7 whether using a denoted object is associated with a movement towards the body (score $=1$ ) or a movement away from the body (score $=7$ ). Objects used with bi-directional movements or objects without a clear action association should be rated with the score 4 . In this manner we wanted to ascertain that participants had a clear action association with critical word stimuli, even in the absence of behavioral data (see procedures below). Participants indeed rated BW stimuli to be related to movements towards the body (mean $=1.99, \mathrm{SD}=0.49$ ) and WW stimuli to be related to movement away from the body (mean $=4.92, \mathrm{SD}=0.65$ ). Independent sample $t$-tests showed that action association scores differed significantly between the conditions $(t(158)=31.89, p=0.001)$. Further one sample $t$-tests showed that both action association scores differed significantly from 4, indicating that objects were associated with a clear, uni-directional movement (BW: $t(79)=36.34, p<0.001$; WW: $t(79=12.58, p<0.001)$.

\subsubsection{Procedure}

During the fMRI Experiment participants lay on their backs inside the scanner Via a mirror mounted to the headcoil, participants were able to see a projection of a computer screen with a resolution of $1024 \times 768$ pixels at the size of $44.5 \mathrm{~cm} \times 33.3 \mathrm{~cm}$ on which lexical stimuli were presented. The viewing distance (eye to center of screen) was $80.0 \mathrm{~cm}$, resulting in an visual angle of $13^{\circ}$ (for the largest lexical letter string $=12$ letters).

Participants were presented with a total of 200 word stimuli in the scanner. Stimuli belonged to one of 3 conditions: (1) Body Words (BW) denoting objects used at the body (e.g., cup, comb), (2) World Words (WW) denoting object used at target locations in the surrounding environment (e.g., key, screwdriver), and (3) pseudowords (PW). The 200 word stimuli comprised 160 critical items (i.e., $80 \mathrm{BW}$ stimuli and $80 \mathrm{WW}$ stimuli), and 40 catch trials (i.e., PW words, see below). The order of stimulus presentation was randomized individually for each participant. All participants saw all word stimuli.

A trial consisted of visual presentation of a single word stimulus (or in the case of Null trials, presentation of a blank screen). At the beginning of each trial a variable jitter time of $0,500,1000$ or $1500 \mathrm{~ms}$ was included, in order to improve the sampling rate of the BOLD signal. Following the jitter, a white fixation cross-appeared on the screen for $500 \mathrm{~ms}$. Directly following the fixation cross a movement-preparation cue (i.e., the letter A or B) was shown for $1000 \mathrm{~ms}$. This was followed directly by the presentation of the stimulus word for $2000 \mathrm{~ms}$. The interstimulus interval was approximately $3 \mathrm{~s}$ (variable due to jitter and response time).

Participants were instructed to read all words carefully, and to perform a go/nogo lexical decision task, in which go responses should be made only in the PW condition. For PW words, participants were instructed to respond as quickly as possible, making the action they had been cued to perform by the preceding movement cue. In this manner, we ensured that participants semantically processed all words (i.e., participants had to comprehend the words in order to decide not to answer), but critical experimental stimuli were kept free of motor execution artifacts.

After participating in the scanning session, participants were asked to rate all of the items they had seen with respect to familiarity, frequency of word use, imageability, action association and the target location to which objects are moved during use.

\subsection{4. fMRI data acquisition}

Functional images were acquired on a Siemens TRIO 3.0 T MRI system (Siemens, Erlangen, Germany) equipped with echo planar imaging (EPI) capabilities, using a birdcage head coil for radio frequency transmission and signal reception. Blood oxygenation level-dependent (BOLD) sensitive functional images were acquired using a single shot gradient EPI-sequence (TE/TR $=30 / 2000 \mathrm{~ms} ; 31$ axial slices in ascending order, voxel size $=3.5 \times 3.5 \times 3.5$ ). High-resolution anatomical images were acquired using a MPRAGE sequence $(\mathrm{TE}=3.03$; voxel size $=1 \mathrm{~mm} \times 1 \mathrm{~mm} \times 1 \mathrm{~mm}, 192$ sagittal slices, FOV $=256$ )

\subsection{5. fMRI data analysis}

Functional data were pre-processed and analyzed with SPM5 (Statistical Parametric Mapping, www.fil.ion.ucl.ac.uk/spm). Preprocessing involved removing the first 3 volumes to allow for T1 equilibration effects. Rigid body registration along 3 translation and 3 rotations was applied to correct for small head movements. Subsequently the time series for each voxel was realigned temporally to acquisition of the middle slice (slice 17), to correct for slice timing acquisition delays. Images were normalized to a standard EPI template centered in MNI space and resampled at an isotropic voxel size of $2 \mathrm{~mm}$. Low-frequency signal changes and base-line drifts were removed by applying a temporal highpass filter to remove frequencies lower than $1 / 120 \mathrm{~Hz}$. The normalized images were smoothed with an isotropic $10 \mathrm{~mm}$ full-width-at-half-maximum (FWHM) Gaussian kernel. The ensuing pre-processed fMRI time series were analyzed on a subject-by-subject basis using an event-related approach in the context of the General Linear Model (GLM) with regressors for each condition (BW, WW, Null) convolved with a canonical hemodynamic response function. The parameters from the motion correction algorithm were included in the model as effects of no interest.

For each participant two contrast images were generated, representing the main effects of body schema representation (Body Word [BW]-Word Word [WW] and WW-BW). Because individual functional datasets had been aligned to the standard stereotactic reference space, a group analysis based on the contrast images could be performed. Single-participant contrast images were entered into a second-level random effects analysis for the critical contrast of interest. The group analysis consisted of a one-sample $t$-test across the contrast images of all subjects that indicated whether observed differences between conditions were significantly distinct from zero. To protect against false positive activation a double threshold was applied, by which only voxels with a $p<0.005$, uncorrected and a volume exceeding 120 voxels $\left(960 \mathrm{~mm}^{3}\right.$ ) were considered (Forman et al., 1995; Slotnick, Moo, Segal, \& Hart, 2003).

\subsection{Results}

\subsubsection{Behavioral results}

Two participants who made more than $85 \%$ errors in any one experimental condition were eliminated from the final analysis. One participant had to be excluded for making excessive movements in the scanner. The remaining 15 participants performed very well in all conditions, indicating that they understood the task and indeed processed all items lexically (mean correct responses $=98.41 \%, \mathrm{SD}=3.27$ ).

\subsubsection{Neuroimaging results}

Areas demonstrating significantly different levels of activation for Body vs. World Words can be seen in Fig. 1 and Table 2. The direct contrast between Body vs. World words showed significantly increased levels of activation in the bilateral extrastriate visual areas, specifically in the middle occipital gyrus extending into the posterior reaches of the middle temporal gyrus. In the left hemisphere activation was very close to what has been described in previous literature as the extrastriate body area (EBA). In the right hemisphere activation was somewhat more posterior and superior to EBA. In addition to the extrastriate visual areas activation was seen to be greater for Body Words than for World Words around the left intraparietal sulcus (IPS), extending into the angular gyrus in the inferior parietal lobe. Furthermore reliably greater activation was seen in the right inferior frontal gyrus (IFG), near the inferior frontal sulus, in the pars opercularis.

The direct contrast between World Words vs. Body Words revealed no significant activations.

\subsubsection{Discussion}

In an fMRI study we investigated the neural correlates of words denoting objects highly associated with movement towards the body (Body Words, such as cup or comb) compared to words denoting objects highly associated with movement away from the body (World Words, such as key or screwdriver). The results indicate that Body Words elicit more activation than World Words in striate and extrastriate visual areas as well as inferior parietal lobe (IPL), and inferior frontal gyrus (IFG). No brain regions were seen to be more activated by World Words than by Body Words. 


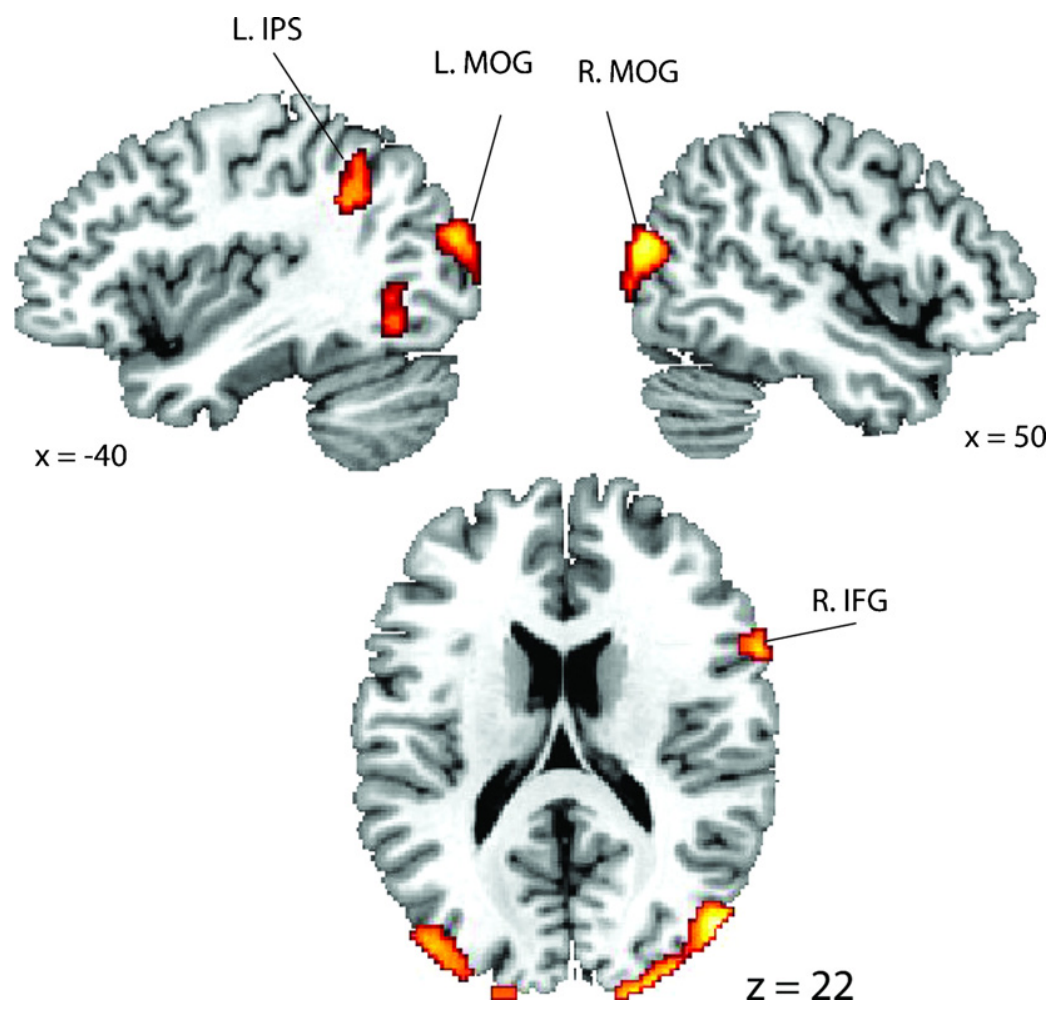

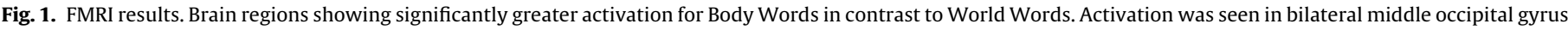

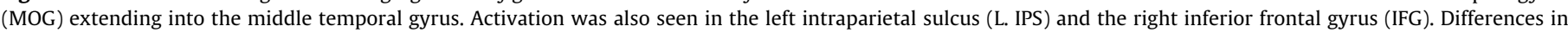
activation passed a double threshold of voxel level $(p<0.005)$ and cluster size $(k>120$ voxels).

In particular the extrastriate visual areas have been described previously in studies investigating the perception of bodily motion (Downing, Jiang, Shuman, \& Kanwisher, 2001; Hodzic, Kaas, Muckli, Stirn, \& Singer, 2009; Taylor, Wiggett, \& Downing, 2007). A region in the posterior portion of the middle temporal gyrus has been dubbed the extrastriate body area (EBA), because it appears to be selectively activated by visual scenes involving human bodies (Downing et al., 2001). This region becomes increasingly activated the more bodyinformation is available to the system (Taylor et al., 2007), and it does not appear to distinguish between agency in body representations (i.e., no difference in activation for observing own vs. someone else's body) (Hodzic et al., 2008; Hodzic, Kaas, et al., 2009; Hodzic, Muckli, Singer, \& Stirn, 2009). Although EBA is commonly described as a visual processing area, it has also been shown to respond to goal-directed movements of body parts in the absence of visual input (Astafiev, Stanley, Shulman, \& Corbetta, 2004). Astafiev and colleagues thus suggest that in addition to its visual recognition function, the EBA integrates relevant visual, spatial and sensory motor signals involved in the representation of the observer's body. Specifically, EBA is postulated to support planning and imagery of movements made with the observer's own body.

In the current study participants were not presented with visual scenes involving bodies, but rather with words denoting objects which are typically used either at a goal location on the body or away from the body. An embodied perspective of language processing suggests that understanding linguistic stimuli (in this case, words) involves simulation of events surrounding the actual physical experience of words' referents. More specifically, processing the word cup will elicit activation in brain areas involved in actually perceiving and handling cups in the physical world. To use any of the objects denoted by Body Words in the current experiment, the denoted object must be brought to a goal location on the body (e.g., in the case of the cup, the functional goal location is the mouth). Therefore, spatial location of specific body areas with respect to one another (i.e., the body schema) is particularly relevant for use of Body Word objects in contrast to World Word objects. Likewise,

Table 2

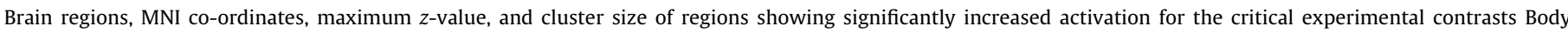
Words > World Words (BW-WW) and World Words > Body Words (WW-BW).

\begin{tabular}{|c|c|c|c|c|c|c|}
\hline Contrast & Brain Region & $x$ & $y$ & $z$ & $z_{\max }$ & Size (voxels) \\
\hline \multirow{11}{*}{ BW-WW } & Occipital lobe & & & & & \\
\hline & Left middle occipital gyrus (IMOG) & -42 & -66 & 2 & 2.94 & 223 \\
\hline & Left middle occipital gyrus & -40 & -86 & 20 & 3.41 & 607 \\
\hline & Right middle occipital gyrus (rMOG) & 50 & -78 & 18 & 4.07 & 439 \\
\hline & Right calcarine gyrus & 14 & -86 & 2 & 3.10 & 219 \\
\hline & Parietal lobe & & & & & \\
\hline & Left intraparietal sulcus (IPS) & -38 & -50 & 32 & 3.07 & 146 \\
\hline & Frontal lobe & & & & & \\
\hline & Right inferior frontal gyrus (IFG) & 64 & 14 & 22 & 3.41 & 121 \\
\hline & Sub-cortical & & & & & \\
\hline & Brain stem & -6 & -22 & -4 & 3.16 & 241 \\
\hline WW-BW & No brain regions with significantly diff & on le & & & & \\
\hline
\end{tabular}


the EBA may be postulated to be particularly involved in supporting planning of movements with Body Word objects, since (1) more body parts are involved in planning a movement with a Body Word object (e.g., hand and mouth for the cup, vs. hand alone for the key), and (2) thus more integration between information coming from different body parts is required (see Taylor et al., 2007). In holding with the embodied language framework, we suggest that increased activation in EBA during lexical-semantic processing of Body Words reflects precisely this.

In addition to EBA, activation in the lateral occipito-temporal region extended posteriorly towards the middle occipital gyrus (MOG). Interestingly, this area is seen to become activated in tasks in which postural control is particularly important (Bakker et al., 2008). Specifically Bakker and colleagues instructed participants to imagine walking along a trajectory in a visual scene. In one condition participants were not substantially restricted in the way in which they could move through the scene. In another condition, participants had to pay close attention to the precise manner in which they placed their feet. This latter condition, in which precise gait control was required, elicited more activation in lateral MOG. The authors suggest that MOG may support generation of visual predictions relevant to motor control, as more precise gait requires more visual updating. As the participant's task was actually a motor imagery and not a motor execution task, it is equally possible that MOG reflects more concentration on postural control relevant for motor control. This would fit nicely with the current paradigm, in which the goal location of Body Word objects can be located by participants only through postural co-ordinates (i.e., we rely more heavily on postural information to find our mouths when drinking from a cup, as the mouth is not generally visible).

In addition to the extrastriate visual areas, increased activation was observed for Body Words in the inferior parietal lobe (IPL), along the banks of the intraparietal sulcus (IPS). The IPL is highly involved in representing one's own body in space. Patients with damage to the right IPL are known to develop disorders of body awareness (e.g., Berlucchi \& Aglioti, 1997). Furthermore, this region is seen to be more active when presented with visual images of one's own vs. another's body (Hodzic, Muckli, et al., 2009). Left IPL has been implicated in coding body parts in imitation tasks (Chaminade, Meltzoff, \& Decety, 2005) and in generating sensorimotor predictions relative to the intended consequences of actions (Bakker et al., 2008). With respect to the current experiment, Body Words, which we argue should be more related than World Words to body information, reliably activated this region. We suggest that this reflects the fact that information about where body parts are in relation to one another (i.e., where one's body parts are in space) is more relevant in functionally using cups and combs (i.e., because the hand must find the mouth to drink, or the head to comb one's hair) than in using keys or pens (i.e., because while information about how to hold the key or pen is crucial to the body, the goal of the action is not body-related). This hypothesis is again supported by the increased involvement of body-relevant brain areas.

Our results thus indicate that the processing of Body Words, in comparison to World Words, reliably activated areas associated with body perception and postural awareness. This is in holding with an embodied perspective of language processing, suggesting that processing words denoting objects reflects real-world experience with using the denoted objects.

Originally we had hypothesized that World Words would elicit more activation than Body Words in visuo-spatial brain areas, since using World Word objects requires detecting the functional goal (e.g., a keyhole when using a key) visually. However, our hypothesis regarding World Words could not be confirmed. This may reflect the fact that functional goals of objects were simply too diverse and thus lacked enough overlap to provide a reliable picture in a group average (i.e., goal locations could be anywhere in the outside world, while functional goals for Body Words tended to be located consistently around one's head). Alternatively it may be the case that Body Words share information about outside goal locations with World Words, since Body Word objects can certainly also be brought to target locations in the world (e.g., a cup can be placed in the sink or brought to the tap). The opposite is not true for World Words (i.e., there are not many cases in which a key should be brought towards one's head). Therefore Body Words may have shared some degree of world representations with World Words, but additionally shown increased reliance on body-relevant areas, while World Words activated no areas selectively in comparison to Body Words.

One further difference between Body Word and World Word objects may be that they engage different frames of reference for spatial processing. Previous research in both animals and humans has shown that processing objects in space makes use of different neural areas depending on whether an egocentric (i.e., object's position is represented in relation to the observer's own body) or an allocentric (i.e., the orientation of the observer is irrelevant) frame of reference is used (Byrne \& Becker, 2008; Neggers, Van der Lubbe, Ramsey, \& Postma, 2006). Specifically, egocentric spatial processing is thought to rely on the visual dorsal stream including superior parietal cortex and premotor areas (Andersen, 1995; Medendorp, Goltz, Crawford, \& Vilis, 2005), while allocentric spatial processing is thought to rely more heavily on the visual ventral stream as well medial temporal areas and the lateral occipital complex (Rolls, 1999). It has been suggested that egocentric spatial processing is more relevant for visuomotor action planning and interactions with objects, while allocentric processing is important for object recognition, memory and cognition (Neggers et al., 2006). In the current experiment, neural responses to words denoting objects used at a location on the body vs. responses to words denoting objects used at locations distinct from the body were investigated. Body Words could thus be construed to be relatively more dependent on an egocentric reference frame (i.e., since objects are brought to locations coded only in a postural co-ordinate system), whereas World Words could be thought to be relatively more dependent on an allocentric reference frame. We see no direct evidence for this distinction in the results of the fMRI study, however this is perhaps not so surprising, since even though the target of Body and World Word objects differ with respect to location, the functional use of both types of objects relies ultimately on egocentric spatial processing, since objects are always manipulated with the observer's own hand. In other words, it is impossible to dissociate between egocentric and allocentric objects, as any object can be used in either an egocentric or an allocentric manner. More specifically, locating an object in space relative to oneself (e.g., by pointing) should rely on egocentric action planning, whereas locating the same object in space relative to other objects (e.g., using a description such as "the key is on the table") should rely relatively more on allocentric action planning.

\section{General discussion}

In two experiments we tested whether implicit body information about how an object is used is activated when processing words denoting these objects. Specifically, participants read words denoting objects typically brought towards the body when used functionally (Body Words, such as cup or comb) and words denoting objects typically brought away from the body when used functionally (World Words, such as key or screwdriver). In a behavioral experiment we found evidence to the effect that preparing a movement congruent to the way in which an object is used facilitates object-word processing. This indicates that action features contribute to lexical-semantic meaning. In a neuroimaging experiment we found evidence for stronger activation in brain regions supporting processing of information about human bodies when 
participants read Body Words in contrast to World Words. This indicates that the functional goal of an object (i.e., the place to which the object must be brought when in use) is also important for lexical-semantic representations. We suggest that Body Words activate body schema to a higher degree than World Words, since the use of Body Word objects relies on postural information to a greater degree than does the use of World Word objects.

The results of the current experiments are in line with an embodied framework of language processing. The behavioral study demonstrates that compatibility effects between action and language processing can be found for single words as well as for sentential propositions (Glenberg \& Kaschak, 2002; see also Myung et al., 2006). The neuroimaging data indicate that brain regions involved in real-world experience with denoted objects become active during language processing. It should be noted here that activation differences were not observed in the sensorimotor areas typically reported in studies looking at the neural correlates of action semantics (i.e., ventral premotor cortex and inferior parietal cortex, see further Chao \& Martin, 2000). In previous studies, the contrast has generally been made between (1) words with an action-semantic component (e.g., manipulable objects, tools) vs. (2) words with no action-semantic component (e.g., non-manipulable objects, animals) (Chao \& Martin, 2000; Saccuman et al., 2006). In the current study however, we contrasted one subgroup of manipulable objects (i.e., Body Words) with a second subgroup of manipulable objects (i.e., World Words). Therefore activation differences were never hypothesized in motor areas, since objects denoted by both word categories should elicit activations consistent with manipulable objects in general.

The neuroimaging results provide new insights into the role of body representations in language processing. Recent investigations have stressed that the human brain is endowed with multiple body representations, which all play different functional roles in action. For instance, the body schema, an egocentric map coding the position of one's body segments in space and time (Head \& Holmes, 1911), has been differentiated from the body structural description, which codes the position of each body segment within a standard body (e.g., Corradi-Dell'Acqua, Tomasino, \& Fink, 2009; Rumiati et al., 2009). These body representations are clearly unique from the classical effector-specific representation of effectors seen in primary and premotor motor cortex (e.g., Rizzolatti \& Luppino, 2001).

While effector-specific representations in primary motor cortex are well documented, locating the neural correlates of other types of body representations requires further investigation. Nevertheless, evidence has been put forth suggesting that body schema may draw on resources of EBA (Astafiev et al., 2004) as well as secondary somatosensory cortex (Corradi-Dell'Acqua et al., 2009). For instance Astafiev and colleagues (2004) reported fMRI evidence that the EBA is strongly modulated by limb movements to visual target stimuli, even in the absence of visual feedback from the movement. Therefore the EBA responds not only during the perception of body parts, but also during goal-directed movements of the observer's own body parts through space.

It seems obvious that body and posture are critical in planning movements and interactions with objects and tools, however less obvious that they should play a role in processing language. Embodied theories of language processing suggest that language is dependent on action simulations to endow words with meaning. With respect to premotor cortex, words denoting actions have been shown to reflect the effector-specificity observed for actual action execution (Hauk, Johnsrude, \& Pulvermüller, 2004; Tettamanti et al., 2005). Taken one step further, if action is relevant to word processing, then those processes relevant to action planning and execution (e.g., body schema) should also become potentially interesting in the language domain. The results of the current neuroimaging study extend the link between language and action to show that other types of action-relevant body representations (i.e., body schema) are also reflected in lexical-semantic representations. Words denoting objects used in a manner requiring relatively more body information (e.g., a cup, since one brings a cup to the mouth-an effector which can only be found by the user using postural co-ordinates) indeed activated brain areas known to be involved in the visual perception of human bodies (EBA) as well as the processing of one's own posture (MOG and IPL).

It is worth noting here that although participants clearly indicated in the post-scanning questionnaire that Body Words are associated with uni-directional movements towards the body, Body Word objects are actually moved as frequently away from the body as they are towards the body in real life. For example, while a cup is brought towards the body for functional use, it is just as frequently brought away from the body once the act of drinking has been completed. This would not appear to be the case for World Word objects, which need never be brought to a location on the actor's own body. Therefore, it is possible that Body Words are associated with more action experience, and thus evoke more intense motor imagery than World Words, resulting in greater neural responses than World Words in the current study. Three points speak against this interpretation, however. First, if Body Word stimuli were more strongly associated with actions in general, we would have expected faster reaction times for Body Words than World Words in our behavioral study. This was not the case (reaction times were close to identical between word conditions). Secondly, the results of the post-scanner questionnaire showed that participant ratings of action associations with words were equally informative for Body and World Words. Lastly, if differences in the neural responses of Body and World Words were a reflection of more vs. less action association in general, we would have expected to observe differences in activation within ventral premotor and inferior parietal cortex, as seen in other studies comparing words with more vs. less action content (Rueschemeyer, Brass, \& Friederici, 2007; Tettamanti et al., 2005). This was not the case. Activation within the cerebral motor system was not significantly different between word conditions.

\section{Conclusion}

The results of two experiments indicate that body schema is reflected in lexical-semantic representations. Body information which is relevant for using an object (i.e., how an object is moved to a given target in space) becomes activated when participants read words denoting objects. The current results extend theories of embodied language processing to show that different types of body representations are reflected in lexical-semantic knowledge.

\section{Appendix A. Supplementary data}

Supplementary data associated with this article can be found, in the online version, at doi:10.1016/j.neuropsychologia.2009.09.019.

\section{References}

Andersen, R. (1995). Encoding of intention and spatial location in the posterior parietal cortex. Cerebral Cortex, 5(5), 457-469.

Arbib, M. A., Bonaiuto, J. B., Jacobs, S., \& Frey, S. H. (2009). Tool use and the distalization of the end-effector. Psychological Research, 73(4), 441-462.

Assmus, A., Giessing, C., Weiss, P., \& Fink, G. R. (2007). Functional interactions during the retrieval of conceptual action knowledge: An fMRI study. Journal of Cognitive Neuroscience, 19(6), 1004-1012.

Astafiev, S., Stanley, C., Shulman, G., \& Corbetta, M. (2004). Extrastriate body area in human occipital cortex responds to the performance of motor actions. Nature Neuroscience, 7(5), 542-548.

Bakker, M., De Lange, F. P., Helmich, R. C., Scheering, R., Bloem, B. R., \& Toni, I. (2008) Cerebral correlates of motor imagery of normal and precision gait. Neurolmage, $41,998-1010$. 
Barsalou, L. (2008). Grounded Cognition. Annual Review of Psychology, 59, 617-645. Beauchamp, M., \& Martin, A. (2007). Grounding object concepts in perception and action: Evidence from fMRI studies of tools. Cortex, 43, 461-468.

Berlucchi, G., \& Aglioti, S. (1997). The body in the brain: Neural bases of corporea awareness. Trends in Neuroscience, 20(12), 560-564.

Borreggine, K. L., \& Kaschak, M. A. (2006). The action-sentence compatibility effect: It's all in the timing. Cognitive Science, 30, 1097-1112.

Boulenger, V., Roy, A., Paulignan, Y., Deprez, V., Jeannerod, M., \& Nazir, T. (2006) Cross-talk between language processes and overt motor behavior in the first 200 msec of Processing. Journal of Cognitive Neuroscience, 18(10), 1607-1615.

Bub, D., Masson, M., \& Cree, G. (2008). Evocation of functional and volumetric gestural knowledge by objects and words. Cognition, 106, 27-58.

Byrne, P., \& Becker, S. (2008). A principle for learning egocentric-allocentric transformation. Neural Computation, 20, 709-737.

Chaminade, T., Meltzoff, A. N., \& Decety, J. (2005). An fMRI study of imitation: Action representation and body schema. Neuropsychologia, 43, 115-127.

Chao, L., \& Martin, A. (2000). Representation of manipulable man-made objects in the dorsal stream. NeuroImage, 12, 478-484.

Corradi-Dell'Acqua, C., Tomasino, B., \& Fink, G. (2009). What is the position of an arm relative to the body? neural correlates of body schema and body structural description. The Journal of Neuroscience, 29(13), 4162-4171.

Downing, P., Jiang, Y., Shuman, M., \& Kanwisher, N. (2001). A cortical area selective for visual processing of the human body. Science, 293, 2470-2473.

Fischer, M., \& Zwaan, R. (2008). Embodied language: A review of the role of the motor system in language comprehension. The Quarterly Journal of Experimental Psychology, 61(6), 825-850.

Forman, S., Cohen, J., Fitzgerald, M., Eddy, W., Mintun, M., \& Noll, D. (1995). Improved assessment of significant activation in functional magnetic resonance imaging (fMRI): Use of a cluster-size threshold. MRM, 33, 636-647.

Glenberg, A. M., \& Kaschak, M. P. (2002). Grounding language in action. Psychonomic Bulletin E' Review, 9, 558-565.

Gonzalez, J., Barros-Loscertales, A., Pulvermüller, F., Meseguer, V., Sanjuan, A., Belloch, V., \& Avilaa, C. (2006). Reading cinnamon activates olfactory brain regions. NeuroImage, 32, 906-912.

Hauk, O., Johnsrude, I., \& Pulvermüller, F. (2004). Somatotopic representation of action words in human motor and premotor cortex. Neuron, 41, 301-307.

Head, H., \& Holmes, G. (1911). Sensory disturbances from cerebral lesion. Brain, 34, $102-254$.

Hodzic, A., Kaas, A., Muckli, L., Stirn, A., \& Singer, W. (2009). Distinct cortical networks for the detection and identification of human body. NeuroImage, 45, 1264-1271.

Hodzic, A., Muckli, L., Singer, W., \& Stirn, A. (2009). Cortical responses to self and others. Human Brain Mapping, 30, 951-962.

Holmes, N. P., \& Spence, C. (2004). The body schema and the multisensory representation(s) of peripersonal space. Cognition Process, 5(2), 94-105.

Iriki, A., Tanaka, M., \& Iwamura Y. (1996). Coding of modified body schema during tool use by macaque postcentral neurones. Neuroreport, 7, 2325-2330.

Maravita, A., Spence, C., \& Driver, J. (2003). Multisensory integration and the body schema: Close to hand and within reach. Current Biology, 13(13), R531-R539.
Maravita, A., \& Iriki, A. (2004). Tools for the body (schema). Trends in Cognitive Sciences, 8(2), 79-86.

Masson, M., Bub, D., \& Newton-Taylor, M. (2008). Language-based access to gestural components of conceptual knowledge. The Quarterly Journal of Experimental Psychology, 61(6), 869-882.

Medendorp, W. P., Goltz, H. C., Crawford, J. D., \& Vilis, T. (2005). Integration of target and effector information in human posterior parietal cortex for the planning of action. Journal of Neurophysiology, 93(2), 954-962.

Myung, J., Blumstein, S., \& Sedivy, J. (2006). Playing on the typewriter, typing on the piano: Manipulation knowledge of objects. Cognition, 98, 223-243.

Neggers, S., Van der Lubbe, R., Ramsey, N., \& Postma, A. (2006). Interactions between ego- and allocentric neuronal representations of space. NeuroImage, $31,320-331$.

Pulvermüller, F. (2001). Brain reflections of words and their meaning. Trends in Cognitive Sciences, 5(12), 517-524.

Rizzolatti, G., \& Luppino, G. (2001). The cortical motor system. Neuron, 31, 889-901.

Rosenbaum, D. A., Loukopoulos, L. D., Meulenbroek, R., Vaughan, J., \& Englebrecht, S. (1995). Planning reaches by evaluating stored postures. Psychological Review, 102, 28-67.

Rueschemeyer, S.-A., Brass, M., \& Friederici, A. D. (2007). Comprehending prehending: Neural correlates of processing verbs with motor stems. Journal of Cognitive Neuroscience, 19(5), 855-865.

Rueschemeyer, S. -A., van Rooij, D., Lindemann, O., Willems R., \& Bekkering, H. (in press). The function of words: Distinct neural correlates for words denoting differently manipulable objects. Journal of Cognitive Neuroscience.

Rumiati, R., Carmo, J., \& Corradi-Dell'Acqua, C. (2009). Neuropsychological perspectives on the mechanisms of imitation. Philosophical Transaction of Royal Society $B, 364(1528), 2337-2347$.

Saccuman, C., Cappa, S., Bates, E., Arevalo, A., Della Rosa, P., Danna, M., \& Perani, D. (2006). The impact of semantic reference on word class: An fMRI study of action and object naming. Neurolmage, 32, 1865-1878.

Slotnick, S. D., Moo, L. R., Segal, J. B., \& Hart, J. (2003). Distinct prefrontal cortex activity associated with item memory and source memory for visual shapes. Cognitive Brain Research, 17, 75-82.

Taylor, J., Wiggett, A., \& Downing, P. (2007). Functional MRI analysis of body and body part representations in the extrastriate and fusiform body areas. Journal of Neurophysiology, 98, 1626-1633.

Tettamanti, M., Buccino, G., Saccuman, M. Gallese, V Danna, M. Scifo, P., Fazio, F. Rizzolatti, G., Cappa, S., \& Perani, D. (2005). Listening to action-related sentences activates fronto parietal motor circuits. Journal of Cognitive Neuroscience, 17(2), 273-281.

Tucker, M., \& Ellis, R. (2004). Action priming by briefly presented objects. Acta Psychologica, 116, 185-203.

Zwaan, R., \& Taylor, L. (2006). Seeing, acting, understanding: Motor resonance in language comprehension. Journal of Experimental Psychology: General, 135(1), $1-11$. 